\title{
MEROMORPHIC UNIVALENT FUNCTIONS WITH NEGATIVE COEFFICIENTS
}

\author{
S. M. SARANGI AND SUGUNA B. URALEGADDI
}

\begin{abstract}
Coefficient inequalities, distortion theorem, extreme points and property preserving integral operators are obtained for certain subclasses of meromorphic starlike functions with negative coefficints. Convolutions of functions in these classes are also obtained.
\end{abstract}

\section{Introduction}

Let $S$ denote the class of functions of the form $f(z)=z+\sum_{n=2}^{\infty} a_{n} z^{n}$ that are analytic and univalent in the unit disk $U=\{z:|z|<1\}$. A function $f \in S$ is said to be starlike of order $\alpha(0 \leq \alpha<1)$, denoted $f \in S^{*}(\alpha)$, if $\operatorname{Re}\left\{z f^{\prime}(z) / f(z)\right\}>\alpha(|z|<1)$ and is said to be convex of order $\alpha$, denoted $f \in K(\alpha)$, if $\operatorname{Re}\left\{1+z f^{\prime \prime}(z) / f^{\prime}(z)\right\}>\alpha(|z|<1)$.

Let $T$ denote the subclass of $S$ consisting of analytic and univalent functions of the form

$$
f(z)=z-\sum_{n=2}^{\infty}\left|a_{n}\right| z^{n} .
$$

Denote by $T^{*}(\alpha)$ and $C(\alpha)$ the subclasses of $T$ that are, respectively starlike of order $\alpha$ and convex of order $\alpha$. In [6] H. Silverman has obtained coefficient inequalities, distortion and covering theorems and extreme points for the classes $T^{*}(\alpha)$ and $C(\alpha)$.

Further let $V$ be the subclass of $S$ consisting of functions of the form $f(z)=z+$ $\sum_{n=2}^{\infty}\left|a_{n}\right| z^{n}$. For $1<\beta \leq 4 / 3$ and $z \in U$, let $V(\beta)=\left\{f \in V: \operatorname{Re}\left(z f^{\prime}(z) / f(z)\right)<\beta\right\}$ and $U(\beta)=\left\{f \in V: \operatorname{Re}\left(1+z f^{\prime \prime}(z) / f^{\prime}(z)\right)<\beta\right\}$. In [8] B.A. Uralegaddi, M.D. Ganigi and S.M. Sarangi examined the classes $V(\beta)$ and $U(\beta)$ and showed that all functions in $V(\beta)$ are starlike and all functions in $U(\beta)$ are convex.

Let $\Sigma$ denote the class of functions $f(z)=\frac{1}{z}+\sum_{n=1}^{\infty} a_{n} z^{n}$ that are regular in the punctured disk $E=\{z: 0<|z|<1\}$ with a simple pole at $z=0$. Let $\Sigma_{s}$ be

Received July 8, 1994.

1991 Mathematics Subject Classification. Primary 30C45.

Key words and phrases. Meromorphic starlike functions, convex functions, convolution. 
the subclass of $\Sigma$ consisting of univalent functions. A function $f \in \Sigma$ is said to be meromorphically starlike of order $\alpha(0 \leq \alpha<1)$, denoted $f \in \Sigma^{*}(\alpha)$ if $\operatorname{Re} z f^{\prime}(z) / f(z)<$ $-\alpha(|z|<1)$ and is said to be meromorphically convex of order $\alpha$, denoted $f \in \Sigma_{k}(\alpha)$, if $\operatorname{Re}\left(1+z f^{\prime \prime}(z) / f^{\prime}(z)\right)<-\alpha(|z|<1) . \Sigma^{*}(0)$ is identified with $\Sigma^{*}$, the class of meromorphic starlike functions and $\Sigma_{k}(0)$ is identified with $\Sigma_{k}$, the class of meromorphic convex functions.

Let $\Sigma_{N}$ denote the subclass of $\Sigma$ consisting of the functions of the form

$$
\begin{aligned}
& f(z)=\frac{1}{z}-\sum_{n=1}^{\infty} a_{n} z^{n}, \quad a_{n} \geq 0 \quad \text { and } \\
& \Sigma_{N}^{*}(\alpha)=\Sigma^{*}(\alpha) \cap \Sigma_{N} \\
& \Sigma_{k, N}(\alpha)=\Sigma_{k}(\alpha) \cap \Sigma_{N}
\end{aligned}
$$

For $1<\beta \leq 2$ let $\Sigma_{N}^{* *}(\beta)$ be the subclass of functions of the form given by (1) satisfying

$$
\operatorname{Re} z f^{\prime}(z) / f(z)>-\beta \text { for }|z|<1 \text {. }
$$

In this paper we obtain coefficient inequalities, distortion Theorem, extreme points and property preserving integral operators for the class $\Sigma_{N}^{* *}(\beta)$. Further it is shown that all functions in $\Sigma_{N}^{* *}(\beta)$ are starlike. Also convolutions of functions in $\Sigma_{N}^{* *}(B)$ are considered.

In [7] B.A. Uralegaddi and M.D. Ganigi and in [3] M.L. Mogra T.R. Reddy and O.P. Juneja have studied mermorphic starlike functions with positive coefficients.

\section{Coefficient Inequalities}

In this section we shall find the necessary and sufficient condition for $f(z)$ to be in $\Sigma_{N}^{* *}(\beta)$.

Theorem 1. Let $f(z)=1 / z+\sum_{n=1}^{\infty} a_{n} z^{n}$ be in $\Sigma$. If $\sum_{n=1}^{\infty}(n+\beta)\left|a_{n}\right| \leq \beta-1$, then $\operatorname{Re}\left(z f^{\prime}(z) / f(z)\right)>-\beta$.

Proof. Let $\sum_{n=1}^{\infty}(n+\beta)\left|a_{n}\right| \leq \beta-1$, It suffices to show that

$$
\left|\frac{z f^{\prime}(z) / f(z)+1}{z f^{\prime}(z) / f(z)-(1-2 \beta)}\right|<1, \quad z \in U .
$$

We have

$$
\begin{aligned}
& \quad\left|\frac{z f^{\prime}(z) / f(z)+1}{z f^{\prime}(z) / f(z)-(1-2 \beta)}\right|=\left|\frac{\sum_{n=1}^{\infty}(n+1) a_{n} z^{n+1}}{2(\beta-1)+\sum_{n=1}^{\infty}(n+2 \beta-1) a_{n} z^{n+1}}\right| \\
& \leq \frac{\sum_{n=1}^{\infty}(n+1)\left|a_{n}\right||z|^{n+1}}{2(\beta-1)-\sum_{n=1}^{\infty}(n+2 \beta-1)\left|a_{n}\right||z|^{n+1}} \\
& \leq \frac{\sum_{n=1}^{\infty}(n+1)\left|a_{n}\right|}{2(\beta-1)-\sum_{n=1}^{\infty}(n+2 \beta-1)\left|a_{n}\right|} .
\end{aligned}
$$


The above expression is bounded by 1 if

$$
\sum_{n=1}^{\infty}(n+1)\left|a_{n}\right| \leq 2(\beta-1)-\sum_{n=1}^{\infty}(n+2 \beta-1)\left|a_{n}\right|
$$

which is equivalent to

$$
\sum_{n=1}^{\infty}(n+\beta)\left|a_{n}\right| \leq \beta-1
$$

But the above inequality is true by hypothesis, the result follows.

For functions in $\Sigma_{N}^{* *}(\beta)$, the converse of theorem 1 is also true.

Theorem 2. A function $f(z)=\frac{1}{z}-\sum_{n=1}^{\infty} a_{n} z^{n}, a_{n} \geq 0$ is in $\Sigma_{N}^{* *}(\beta)$ if and only if $\sum_{n=1}^{\infty}(n+\beta) a_{n} \leq \beta-1$.

Proof. In view of theorem 1, it suffices to show the only if part. Suppose

$$
\operatorname{Re} z f^{\prime}(z) / f(z)=\operatorname{Re} \frac{-1-\sum_{n=1}^{\infty} n a_{n} z^{n+1}}{1-\sum_{n=1}^{\infty} a_{n} z^{n+1}}>-\beta, z \in U
$$

Choose values of $z$ on the real axis so that $z f^{\prime}(z) / f(z)$ is real. Upon clearing the denominator in (2) and letting $z \rightarrow 1$ through real values, we get

$$
1+\sum_{n=1}^{\infty} n a_{n} \leq \beta\left(1-\sum_{n=1}^{\infty} a_{n}\right)
$$

Thus we have $\sum_{n=1}^{\infty}(n+\beta) a_{n} \leq \beta-1$.

Corollary. If $f \in \Sigma_{N}^{* *}(\beta)$ then $a_{n} \leq \frac{\beta-1}{n+\beta}$, with equality only for functions of the form $f_{n}(z)=\frac{1}{z}-\frac{\beta-1}{n+\beta} z^{n}$.

\section{Distortion theorem}

Using coefficient inequalities we shall prove

Theorem 3. If $f \in \Sigma_{N}^{* *}(\beta)$ then

$$
\frac{1}{r}-\frac{\beta-1}{1+\beta} r \leq|f(z)| \leq \frac{1}{r}+\frac{\beta-1}{1+\beta} r \quad(|z|=r)
$$

with equality for $f(z)=\frac{1}{z}-\frac{\beta-1}{1+\beta} z . \quad(z=i r, r)$

Proof. From theorem 2 we have $(1+\beta) \sum_{n=1}^{\infty} a_{n} \leq \sum_{n=1}^{\infty}(n+\beta) a_{n} \leq \beta-1$. Thus $\sum_{n=1}^{\infty} a_{n} \leq \frac{\beta-1}{1+\beta}$ and $|f(z)| \leq \frac{1}{r}+\sum_{n=1}^{\infty} a_{n} r^{n} \leq \frac{1}{r}+r \sum_{n=1}^{\infty} a_{n} \leq \frac{1}{r}+\frac{\beta-1}{1+\beta} r$. Also $|f(z)| \geq \frac{1}{r}-\sum_{n=1}^{\infty} a_{n} r^{n} \geq \frac{1}{r}-r \sum_{n=1}^{\infty} a_{n} \geq \frac{1}{r}-\frac{\beta-1}{1+\beta} r$ Thus the result follows. 


\section{Order of Starlikeness}

Theorem 4. If $f \in \Sigma_{N}^{* *}(\beta)$ then $f \in \Sigma_{N}^{*}(2-\beta)$.

Proof. Suppose $f \in \Sigma_{N}^{* *}(\beta)$. Then $\sum_{n=1}^{\infty}(n+\beta) a_{n} \leq \beta-1$. Sufficient condition for $f$ to be in $\Sigma^{*}(\alpha)$ is $\sum_{n=1}^{\infty}(n+\alpha)\left|a_{n}\right| \leq 1-\alpha$. We must prove that

$$
\sum_{n=1}^{\infty} \frac{n+\beta}{\beta-1} a_{n} \leq 1 \text { implies } \sum_{n=1}^{\infty} \frac{n+(2-\beta)}{1-(2-\beta)}\left|a_{n}\right| \leq 1 .
$$

That is we have to show that

$$
\frac{n+\beta}{\beta-1} \geq \frac{n+2-\beta}{1-2+\beta} \quad{ }^{\prime}=1,2, \ldots
$$

That is equavalent to $(\beta-1) \geq 0$, which is obvious.

Remark. From Theorem 4 it follows that all functions in $\Sigma_{N}^{* *}(\beta)$ are starlike hence univealent.

\section{Extreme Points}

In view of Theorem 2 , the class $\Sigma_{N}^{* *}(\beta)$ is closed under convex linear combinations. We shall determine the extreme points for $\Sigma_{N}^{* *}(\beta)$.

Theorem 5. Let $f_{0}(z)=\frac{1}{z}$ and $f_{n}(z)=\frac{1}{z}-\frac{\beta-1}{n+\beta} z^{n}$ for $n=1,2, \ldots$. Then $f \in \Sigma_{N}^{* *}(\beta)$ if and only if it can be expressed in the form $f(z)=\sum_{n=0}^{\infty} \lambda_{n} f_{n}(z)$ where $\lambda_{n} \geq 0$ and $\sum_{n=0}^{\infty} \lambda_{n}=1$.

Proof. Suppose $f(z)=\sum_{n=0}^{\infty} \lambda_{n} f_{n}(z)=\frac{1}{z}-\sum_{n=1}^{\infty} \lambda_{n} \frac{\beta-1}{n+\beta} z^{n}$, then

$$
\sum_{n=1}^{\infty} \lambda_{n} \frac{\beta-1}{n+\beta} \frac{n+\beta}{\beta-1}=\sum_{n=1}^{\infty} \lambda_{n}=1-\lambda_{0} \leq 1
$$

Hence $f \in \Sigma_{N}^{* *}(\beta)$.

Conversly suppose $f(z) \in \Sigma_{N}^{* *}(\beta)$, then we have

$$
a_{n} \frac{\beta-1}{n+\beta} . \quad n=1,2, \cdots .
$$

Taking $\lambda_{n}=\frac{n+\beta}{\beta-1} a_{n}, n=1,2, \ldots$ and $\lambda_{0}=1-\sum_{n=1}^{\infty} \lambda_{n}$ we have $f(z)=\sum_{n=0}^{\infty} \lambda_{n} f_{n}(z)$

Corollary. The extreme points of $\Sigma_{N}^{* *}(\beta)$ are the functions $f_{n}(z), n=$ $0,1,2 \ldots$ 


\section{Integral Operators}

In this section we shall consider integral operators of the form considered by S.K. Bajpai [1] and R. M. Goel and N.S. Sohi [2].

Theorem 6. If $f(z)=\frac{1}{z}-\sum_{n=1}^{\infty} a_{n} z^{n}, a_{n} \geq 0$ is in $\Sigma_{N}^{* *}(\beta)$, then $F(z)=$ $\frac{c}{z^{c+1}} \int_{0}^{z} t^{c} f(t) d t=\frac{1}{z}-\sum_{n=1}^{\infty} \frac{c}{c+n+1} a_{n} z^{n}, c>0$ belongs to $\Sigma_{N}^{* *}(\gamma)$ where $\gamma=\frac{1+\beta+\beta c}{1+\beta+c}$. The result is sharp for $f(z)=\frac{1}{z}-\frac{\beta-1}{1+\beta} z$.

Proof. Suppose $f(z)=\frac{1}{z}-\sum_{n=1}^{\infty} a_{n} z^{n} \in \Sigma_{N}^{* *}(\beta)$. We have $F(z)=\frac{1}{z}-\sum_{n=1}^{\infty} \frac{c}{c+n+1} a_{n} z^{n}$. It suffices to show that

$$
\sum_{n=1}^{\infty} \frac{n+\gamma}{\gamma-1} \frac{c}{c+n+1} a_{n} \geq 1
$$

Since $f(z) \in \Sigma_{N}^{* *}(\beta)$, we have $\sum_{n=1}^{\infty} \frac{n+\beta}{\beta-1} a_{n} \leq 1$. Hence (3) will be satisfied if $\frac{(n+\gamma) c}{(\gamma-1)(c+n+1)} \leq \frac{n+\beta}{\beta-1}$. Solving for $\gamma$ we obtain

$$
\gamma \geq \frac{n+\beta+\beta c}{n+\beta+c}
$$

Since the right hand side of the above inequality (4) is decreasing function of $n$, putting $n=1$ in (4)

$$
\gamma \geq \frac{1+\beta+\beta c}{1+\beta+c}
$$

Hence the result.

Next we shall consider the converse of theorem 6 .

Theorem 7. Let $F(z)=1 / z-\sum_{n=1}^{\infty} a_{n} z^{n}, a_{n} \geq 0$ be in $\Sigma_{N}^{* *}(\beta)$ and $f(z)=$ $\frac{1}{c}\left[(c+1) F(z)+z F^{\prime}(z)\right]=\frac{1}{z}-\sum_{n=1}^{\infty} \frac{c+n+1}{c} a_{n} z^{n}, c>0$. Then Re $z f^{\prime}(z) / f(z)>-\gamma$, $(1<\gamma \leq 2)$ for $0<|z|<r(\beta, \gamma)$. Where $r(\beta, \gamma)=\inf \left\{\frac{c(n+\beta)(\gamma-1)}{(c+n+1)(\beta-1)(n+\gamma)}\right\}^{1 / n+1}$, $n=1,2, \ldots$ The result is sharp for $F(z)=\frac{1}{z}-\frac{\beta-1}{n+\beta} z^{n}$ for some $n$.

Proof. It suffices to show that

$$
\left|\frac{z f^{\prime}(z) / f(z)+1}{z f^{\prime}(z) / f(z)-(1-2 \gamma)}\right|<1 \text { for } 0<|z|<r(\beta, \gamma)
$$

We have

$$
\left|\frac{z f^{\prime}(z) / f(z)+1}{z f^{\prime}(z) / f(z)-(1-2 \gamma)}\right| \leq \frac{\sum_{n=1}^{\infty}\left[\frac{c+n+1}{c}\right](n+1) a_{n}|z|^{n+1}}{2(\gamma-1)-\sum_{n=1}^{\infty} \frac{c+n+1}{c}(n+2 \gamma-1) a_{n}|z|^{n+1}}
$$


This expression is bounded by 1 if

$$
\sum_{n=1}^{\infty}\left[\frac{c+n+1}{c}\right](n+1) a_{n}|z|^{n+1} \leq 2(\gamma-1)-\sum_{n=1}^{\infty}\left[\frac{c+n+1}{c}\right](n+2 \gamma-1) a_{n}|z|^{n+1}
$$

which is equivalent to

$$
\sum_{n=1}^{\infty}\left[\frac{c+n+1}{c}\right] \frac{(n+\gamma)}{\gamma-1} a_{n}|z|^{n+1} \leq 1
$$

But from Theorem (2) we have $\sum_{n=1}^{\infty} \frac{n+\beta}{\beta-1} a_{n} \leq 1$.

Hence (5) will be satisfied if

$$
\frac{c+n+1}{c} \frac{n+\gamma}{\gamma-1}|z|^{n+1} \leq \frac{n+\beta}{\beta-1}
$$

Solving for $|z|$ we get

$$
|z| \leq\left\{\frac{c(n+\beta)(\gamma-1)}{(c+n+1)(\beta-1)(n+\gamma)}\right\}^{1 / n+1}, \quad n=1,2, \ldots
$$

Writing $|z|=r(\beta, \gamma)$ in (6) the result follows.

We have

$$
\begin{aligned}
r(\beta, \beta) & =\inf \left\{\frac{c}{c+n+1}\right\}^{1 / n+1}, n=1,2, \ldots \\
& =(c / c+2)^{1 / 2}
\end{aligned}
$$

\section{Convolution Properties}

In [4] M.S. Robertson has shown that if $f(z)=1 / z+\sum_{n=1}^{\infty} a_{n} z^{n}$ and $g(z)=$ $1 / z+\sum_{n=1}^{\infty} b_{n} z^{n}$ are in $\Sigma_{s}$ then their convolution $f(z) * g(z)=1 / z+\sum_{n=1}^{\infty} a_{n} b_{n} z^{n}$ is also in $\Sigma_{s}$.

Here we shall prove the following results for functions in $\Sigma_{N}^{* *}(\beta)$. Methods used are similar to those in [5].

Theorem 8. If $f(z)=1 / z-\sum_{n=1}^{\infty} a_{n} z^{n}, a_{n} \geq 0$ and $g(z)=1 / z-\sum_{n=1}^{\infty} b_{n} z^{n}$, $b_{n} \geq 0$ are members of $\Sigma_{N}^{* *}(\beta)$, then $h(z)=f(z) * g(z)=1 / z-\sum_{n=1}^{\infty} a_{n} b_{n} z^{z}$ is a member of $\Sigma_{N}^{* *}(\gamma)$ where $\gamma=\frac{\beta^{2}+1}{2 \beta}$.

Proof. Since $f(z)$ and $g(z)$ belong to $\Sigma_{N}^{* *}(\beta)$ we have from Theorem 2

$$
\sum_{n=1}^{\infty}(n+\beta) a_{n} \leq \beta-1 \text { and } \sum_{n=1}^{\infty}(n+\beta) b_{n} \leq \beta-1 .
$$


To show that $h(z) \in \Sigma_{N}^{* *}(\gamma)$, we have to show

$$
\sum_{n=1}^{\infty}(n+\gamma) a_{n} b_{n} \leq \gamma-1
$$

Evidently, we want to shwo that

$$
\begin{aligned}
& \sum_{n=1}^{\infty} \frac{n+\beta}{\beta-1} a_{n} \leq 1 \quad \text { and } \\
& \sum_{n=1}^{\infty} \frac{n+\beta}{\beta-1} b_{n} \leq 1
\end{aligned}
$$

imply that

$$
\sum_{n=1}^{\infty} \frac{n+\gamma}{\gamma-1} a_{n} b_{n} \leq 1
$$

From (7) and (8), we get by means of Cauchy - Schwarz inequality

$$
\sum_{n=1}^{\infty} \frac{n+\beta}{\beta-1} \sqrt{a_{n} b_{n}} \leq 1
$$

It is therefore enough to prove that

$$
\begin{gathered}
\frac{n+\gamma}{\gamma-1} a_{n} b_{n} \leq \frac{n+\beta}{\beta-1} \sqrt{a_{n} b_{n}} \quad n=1,2, \ldots \\
\text { i.e. } \quad \sqrt{a_{n} b_{n}} \leq \frac{n+\beta}{\beta-1} \frac{\gamma-1}{n+\gamma}
\end{gathered}
$$

From (9) it follows that

$$
\sqrt{a_{n} b_{n}} \leq \frac{\beta-1}{n+\beta} \text { for each } n
$$

Therefore it will be sufficient to prove that

$$
\frac{\beta-1}{n+\beta} \leq \frac{n+\beta}{\beta-1} \frac{\gamma-1}{n+\gamma} \text { for all } n
$$

Solving for $\gamma$, we obtain

$$
\gamma \geq \frac{(n+\beta)^{2}+n(\beta-1)^{2}}{(n+\beta)^{2}-(\beta-1)^{2}}
$$

The right hand side of the above inequality is decresing function of $n(n=1,2, \ldots)$. Therefore setting $n=1$ in (10) we get

$$
\gamma \geq \frac{\beta^{2}+1}{2 \beta}
$$


The result is sharp, with equality when $f(z)=g(z)=\frac{1}{z}-\frac{\beta-1}{1+\beta} z$

Theorem 9. If $f(z) \in \Sigma_{N}^{* *}(\beta)$ and $g(z) \in \Sigma_{N}^{* *}(\gamma)$ then $f(z) * g(z) \in \Sigma_{N}^{* *}(\delta)$ where $\delta=\frac{\beta \gamma+1}{\beta+\gamma}$

Proof. Proceeding as in the proof of the above theorem, we get

$$
\delta \geq \frac{1+n\left[\begin{array}{ll}
\frac{\beta-1}{n+\beta} & \left.\frac{\gamma-1}{n+\gamma}\right]
\end{array}\right]}{1-\frac{\beta-1}{n+\beta} \frac{\gamma-1}{n+\gamma}}
$$

Right hand side of (11) in decreasing function of $n$.

Therefore putting $n=1$ in (11), we get

$$
\delta \geq \frac{\beta \gamma+1}{\beta+\gamma}
$$

Corollary. If $f(z), g(z), h(z) \in \Sigma_{N}^{* *}(\beta)$ then $f * g * h \in \Sigma_{N}^{* *}\left[\frac{\beta^{3}+3 \beta}{3 \beta^{2}+1}\right]$.

Proof. From Theorem 8 we have $f * g \in \Sigma_{N}^{* *}\left[\frac{\beta^{2}+1}{2 \beta}\right]$. Using Theorem 9

$$
(f * g) * h \in \Sigma_{N}^{* *}\left[\frac{\frac{\beta^{2}+1}{2 \beta}+1}{\frac{\beta^{2}+1}{2 \beta}+\beta}\right]=\Sigma_{N}^{* *}\left[\frac{\beta^{3}+3 \beta}{3 \beta^{2}+1}\right] .
$$

\section{References}

[1] S. K. Bajpai, "A note on a class of meromorphic univalent functions," Rev. Roumanie Math. Pures Appl., 22(1977), 295-297.

[2] R. M. Goel and N. S. Sohi, "On a class of meromorphic functions," Glasnik Matematicki, 17(1981), 19-28.

[3] M. L Mogra, T. R. Reddy and O. P. Juneja, "Meromorphic univalent functions with positive coefficients," Bull. Austral. Math. Soc., 32(1985), 161-176.

[4] M. S. Robertson, "Convolutions of Schlicht functions," Proc. Amer. Math. Soc. 13(1962), 585-589.

[5] A Schild and H. Silverman, "Convolution of univalent functions with negative coefficients," Ann. Univ. Mariae. Curie. Sklodwska Sect. A 29(1975), 99-107.

[6] H. Silverman, "Univalent functions with negative coefficients," Proc. Amer. Math. Soc. 51(1975), 109-116.

[7] B. A. Uralegaddi and M. D. Ganigi, "A certain class of mermorphically starlike functions with positive coefficients," Pure and Applied Mathematika Sciences 26(1987), 75-81.

[8] B. A. Uralegaddi, M. D. Ganigi and S. M. Sarangi "Univalent functions with positive coefficients," (To appear in Tamkang J. Maths 25(1994)).

Department of Mathematics, Karnatak University, Dharwad 580 003, India. 